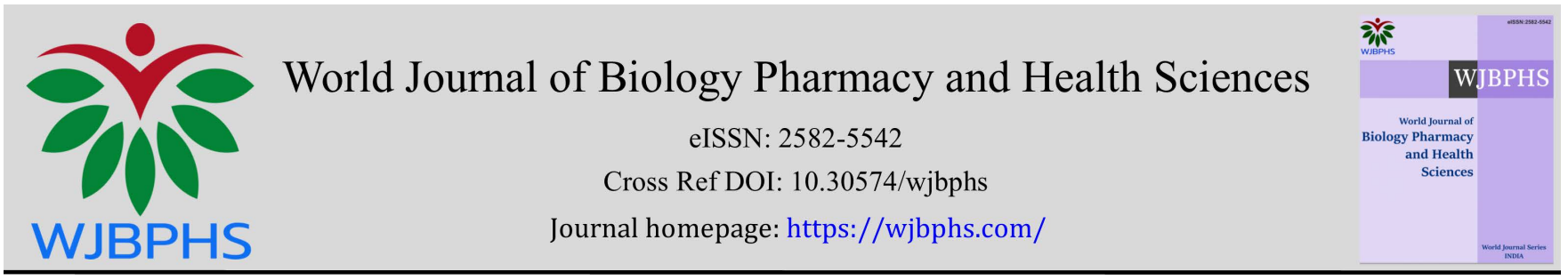

(REVIEW ARTICLE)

\title{
Botulinum toxin in neurological clinical practice
}

\author{
Nusrat Aziz * \\ Department of Physiology, Faculty of Medicine, Umm Al-Qura University, Makkah 24382, Kingdom of Saudi Arabia.
}

World Journal of Biology Pharmacy and Health Sciences, 2021, 05(03), 019-023

Publication history: Received on 23 January 2021; revised on 27 February 2021; accepted on 02 March 2021

Article DOI: https://doi.org/10.30574/wjbphs.2021.5.3.0018

\begin{abstract}
Botulinum toxin is produced by Clostridium botulini bacteria. It has been increasingly used as a boon in medical practice for chemodenervation in conditions like skeletal muscle spasticity, glandular hypersecretion, neuropathic pain and smooth muscle hyperactivity.

The action of Botulinum neurotoxin (BoNT) is by blocking the neurotransmission at the cholinergic nerve endings, by inhibiting the docking and fusion of Acetyl Choline (Ach) vesicles for exocytosis into the synaptic area.Botulinum toxin has been used for management of neurological disorders with great patient satisfaction and appreciation. The effect of botulinum toxin wears off in 3-6 months and may require another dose. Frequent dosing may lead to production of antibodies against BoNT with consequent irresponsiveness to therapy in a few cases.

Scrupulous use of botulinum toxin in the hands of experts may help a long way in giving much needed relief and respite to neurological patients and increase their quality of life. Its use in cosmetic dermatology in reducing wrinkles, by relaxing the facial muscles is very popular
\end{abstract}

Keywords: Botulinum toxin; Botox; spasticity; Neuropathic pain; Hyperhydrosis

\section{Introduction}

Botulinum toxin is produced by the bacteria Clostridium botulinum. This is a potent biological toxin and can produce fatal disease. The spores of Clostridium botulinum are found in soil and can be present in contaminated food. The effect of clostridium botulinum was described by German scientist Justinus Kerner from the outbreaks caused due to bad sausages between1817-1822 and gave an idea of its scope for therapeutic use. These outbreaks typically showed symptoms like blurry vision, drooping eyelids, slurred speech, difficulty swallowing, vomiting, and severe muscle weakness. He described this as the 'fat poison' or 'sausage poison' and even demonstrated its effect by applying on smaller animals. There was paralysis of that group of muscles but showed complete recovery in the next a few days [1]. The toxin of clostridium botulinum was later discovered by Emile Pierre van Ermengemm in 1895 [2] and coined the term Botulus (latin word for sausage). Botulinum toxin was first isolated in crystalline form by Dr. Edward Schantz. Alan B. Scott and Edward Schantz used botulinum toxin in early 1970s as medical treatment for correction of strabismus by relaxing the muscles [3]. They used injection of type A botulinum toxin which was later approved by Food and Drug Administration in 1989. The scientists were focused on studying its effect on hyperactive eye muscles and blepharospasm when they accidently were brought to notice about its effects on forehead wrinkles. Alastair Carruthers and his wife Jean Carruthers first reported the use of botulinum toxin for cosmetic use [4] Since then, FDA has approved the use of Botox for various procedures such as Cervical dystonia in 2000, Glabellar lines in 2002, Axillary hyperhidrosis (excessive sweating) in 2004, Chronic migraines and upper lip spasticity in 2010, Urinary incontinence in 2011 and

\footnotetext{
* Corresponding author: Nusrat Aziz

Department of Physiology, Faculty of Medicine, Umm Al-Qura University, Makkah 24382, Kingdom of Saudi Arabia. 
Crow's feet (lateral canthal lines) in 2013 [5]. Some of the therapeutic indicators for botulinum toxin are given in table 1.

Table 1 Conditions commonly treated by Botulinum toxin

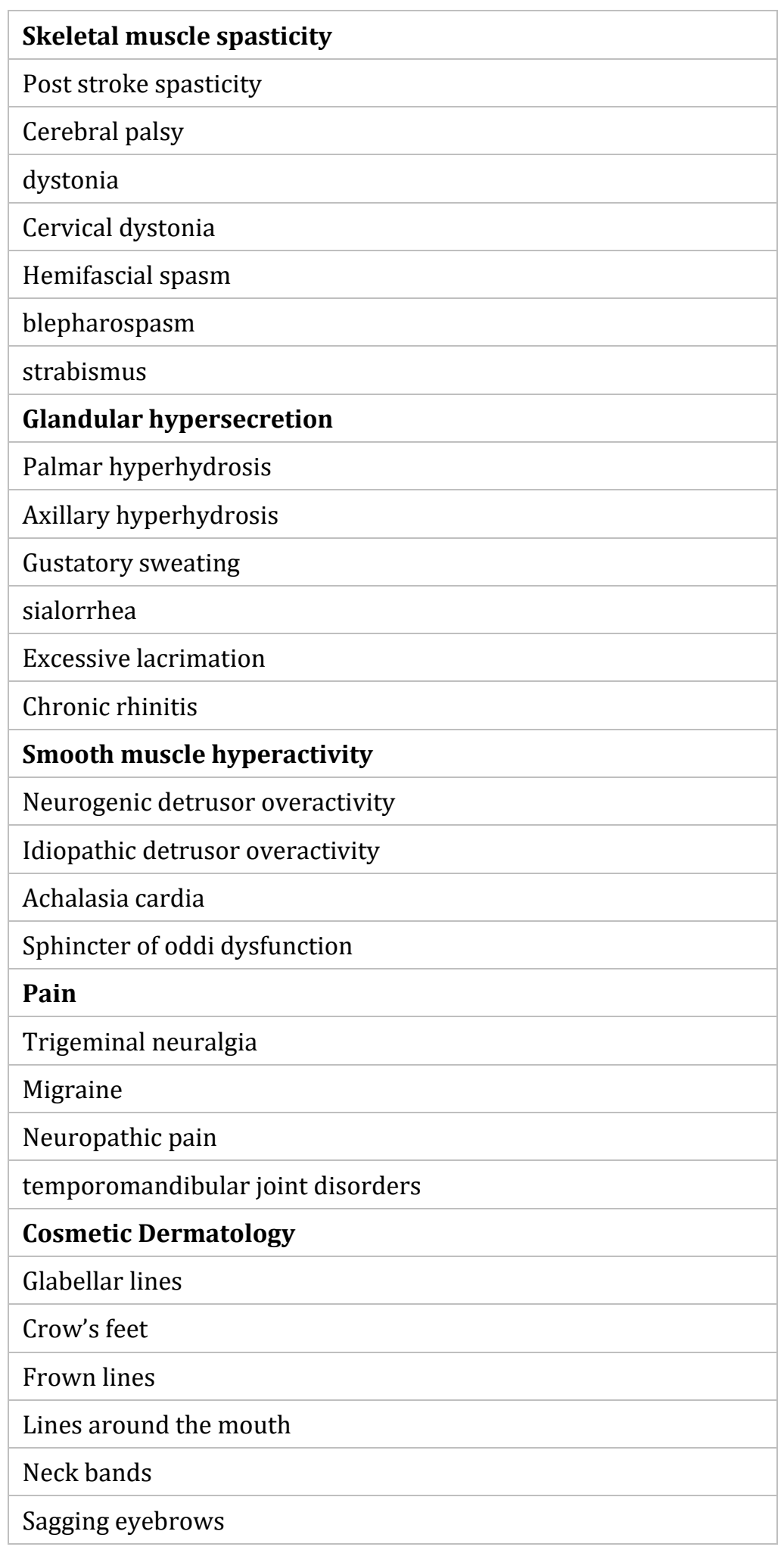




\section{Structure}

Botulinum neurotoxin (BoNT) structurally is made of a heavy protein chain and a light heavy chain bounded with disulphide bond having a molecular weight of $150 \mathrm{kDa}$. It is activated when cleaved into $100 \mathrm{kDa}$ and $50 \mathrm{kDa}$ heavy and light chains respectively. It is present in 7 subtypes A, B, C, D, E, F and G of which subtype A and B are used in therapeutic practice as BoNT-A and BoNT-B are most commonly used.

\section{Mechanism of action}

Botulinum toxin breaks soluble N-ethylmaleimide-sensitive factor attachment protein receptor (SNARE) proteins needed for docking and fusion of vesicles with cell membrane. When botulinum is injected, it binds to ganglioside receptors on the membrane of cholinergic nerve endings by its heavy chains and gets internalized. Once inside the cell it breaks into $50 \mathrm{kDa}$ light chain and $100 \mathrm{kDa}$ heavy chain. BoNT binds to SNARE protein complex by its light chain and cleaves it. The SNARE proteins complex is composed of SNAP 25, Syntaxin and Synaptobrevin needed for docking and fusion at the cell membrane for exocytosis and release of Ach. The mechanism of action of BoNT -A involves cleavage of SNAP 25 whereas the BoNT-B cleaves the synaptobrevin a member of vesicle associated membrane proteins (VAMP) needed for docking of synaptic vesicle to the target cell membrane. Thus, it blocks the docking and fusion process of acetyl choline containing vesicles and therefore the release of neurotransmitter acetyl choline by exocytosis at the neuromuscular junction or the gland is blocked as is shown Fig1 below.

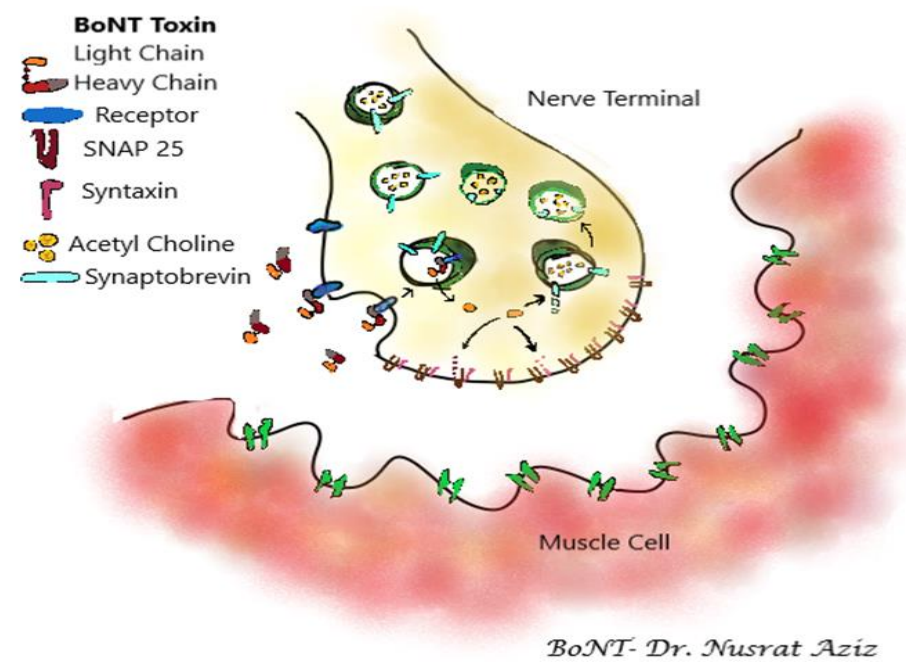

Figure 1 Mechanism of action of Botulinum Neurotoxin (BoNT)

The decreased release of Ach decreases transmission of impulse to the motor end plate and therefore decreases depolarization and contraction of muscle, prevents contraction and promotes relaxation of muscle or blocks secretion at the level of glands. This mechanism is used for the relief of excessive contraction such as in spasm, dystonia and spastic conditions of muscles. It has also been beneficial in movement disorders such as tremors. Chemo denervation with botulinum is also very effective in relief of chronic pain such as in migraine and neuralgia [6] as botulinum toxin also interferes with the release of pain and inflammatory mediators including substance P, glutamate and calcitonin gene related protein from sensory nerve endings and dorsal root ganglion [7]. The action of BoNT at the cholinergic muscarinic ending at sweat gland has been used effectively for treatment of hyperhidrosis [8], sialorrhea [9]. BoNT therapy has shown good results for urinary bladder detrusor overactivity helping patients to lower dosage of systemic anticholinergics [10]. It is used for relaxation of sphincters such as in achalasia cardia [11], sphincter of oddi dysfunction [12], detrusor muscle dyssynergia [13] etc.

Its effect starts in 2-7 days after injection and lasts for 3 to 6 months when another dose can be given. The relaxation of target skeletal muscle can be attributed to decreased activity of alpha motor neurons and also gamma motor neurons. Gamma motor neurons responsible for stretch reflex, are inhibited directly through action on cholinergic nerve endings and also indirectly because of inhibition of alpha motor neuron through alpha gamma coactivation process [14]. The paralyzing effect of BoNT disappears by 3-6months. Termination of activity of botulinum toxin occurs by 1 . Aneural stage showing profuse network of axonal sprouts can be observed with intact function of SNARE proteins and exocytosis 
of Acetyl choline within few days with good muscle response by 28 days 2. Neural stage with reinnervation and stabilization of neuromuscular junction and increase in Ach-receptors in about 3-6 months. [15, 16]. Axonal sprouts are promoted by release of IGF-1 and other neurotrophins exerting their effect on the nerve terminal. Repeated use may lead to development of antibodies against botulinum toxin and can lead to unresponsiveness to therapy. Meta-analysis by Neuman et al showed $0.49 \%$ of individuals being treated with BoNT for multiple disorders showed the conversion of antibody negative status for botulinum antibodies to antibody positive status which was quite low. Maximum percentage of antibody positive status was found in those undergoing therapy for cervical dystonia which was $1.28 \%$. [17]. It can be avoided by using lesser dose, increasing the time duration between doses or changing the type of BoNT used.

\section{Side effects}

The complications of botulism can be related to dosing, frequency, faulty technique, lack of accurate knowledge of anatomical structures, wrong muscle, injection into blood vessel and pre-existing underlying co-morbidities making them more susceptibility to the unwanted effects of BoNT. Headache, bruising, swelling, pain at the site of injection, droopy angle of mouth, eye dryness, unequal level of eyebrows, diplopia and ptosis are some of the side effects of BoNT [18] on face for cosmetic use, blepharospasm and strabismus. Botulism with symptoms like dizziness, fatigue, blurred vision, weakness or paralysis, dysarthria, dysphonia, dysphagia, irregular heartbeat and respiratory arrest might be observed in few cases [19] Death due to anaphylactic shock or Botulism can occur can also occur. Other rare effects include necrotizing fasciitis, pseudoaneurysm of temporal artery, cervical kyphosis, blurred vision, loss of bladder control. [20] Lethal dose of BoNT has been estimated to be about 2500-3000 units in a 70Kg adult [21,22]. Any side effects should be carefully dealt with. Botulism may even require administration of antitoxin.

Pre -existing conditions like myasthenia gravis, motor neuron disease, Lambert eaton syndrome are contraindicated as it may further increase muscle weakness. Hypersensitivity should be kept in mind and should be checked for. It is not recommended in pregnant and breast-feeding women for safety concerns.

Drug interactions with botulinum toxin: Concomitant use of some drugs potentiates or decrease the effect of BoNT which must be looked into before giving injection of BoNT. Aminoglycosides, potentiate the effect of BoNT, whereas use of acetyl cholinesterase inhibitors decrease the effect of BoNT.

\section{Conclusion}

Botulinum toxin effect on decreasing spasticity, pain and glandular functions along with its cosmetic used should be utilized more in improving individual's quality of life by giving respite from suffering. Avoidance of higher doses and not giving very frequently can prevent the side effects and also decrease the chances of developing antibodies against the BoNT. The principle mechanism of action of botulinum toxin that is chemodenervation can be utilized in several disorders involving nerves and their release of neurotransmitters and more and more research should be encouraged for its utility. The neurotoxin derived from the disease-causing clostridium botulini when used in a proficient way in small doses can produce surprising results in medical practice and helping mankind in a long way.

\section{Compliance with ethical standards}

\section{Acknowledgments}

I would like to thank to my husband Fazal Mustafa Ali Khan and my kids for their support and encouragement in fulfilling this project.

\section{Disclosure of conflict of interest}

I declare there is no conflict of interest and no financial aid was taken for this project.

\section{References}

[1] Erbguth FJ, Naumann M. Historical aspects of botulinum toxin: Justinus Kerner (1786-1862) and the sausage poison. Neurology. 1999; 53(8): 1850-1853.

[2] Erbguth FJ. Historical notes on botulism, Clostridium botulinum, botulinum toxin, and the idea of the therapeutic use of the toxin. Mov. Disord. 2004; 19: S2-S6. 
[3] Erbguth FJ. From poison to remedy: the chequered history of botulinum toxin. J Neural Transm (Vienna). 2008; 115(4): 559-565.

[4] Carruthers A, Kiene K, Carruthers J. Botulinum A exotoxin use in clinical dermatology. Journal of the American Academy of Dermatology. 1996; 34(5): 788-797.

[5] The Remarkable Story of Botox [Internet]. Verywell Health. 2021 [cited 2 March 2021]. Available from: https://www.verywellhealth.com/how-botox-came-to-be-1124145

[6] Ramachandran R, Yaksh TL. Botulinum toxins as a prophylaxis for migraine. Br J Pharmacol. 2014; 171: 41774192.

[7] Park J, Park H. Botulinum Toxin for the Treatment of Neuropathic Pain. Toxins. 2017; 9(9): 260.

[8] Lakraj A, Moghimi N, Jabbari B. Hyperhidrosis: Anatomy, Pathophysiology and Treatment with Emphasis on the Role of Botulinum Toxins. Toxins. 2013; 5(4): 821-840.

[9] Hosp C, Naumann MK, Hamm H. Botulinum Toxin Treatment of Autonomic Disorders: Focal Hyperhidrosis and Sialorrhea. Semin Neurol. 2016; 36(1): 20-28.

[10] Zhu Q, Liu J, Yang C. Clinical Study on Combined Therapy of Botulinum Toxin Injection and Small Balloon Dilation in Patients with Esophageal Achalasia. Digestive Surgery. 2009; 26(6): 493-8.

[11] Wehrmann T, Schmitt TH, Arndt A, Lembcke B, Caspary WF, Seifert H. Endoscopic injection of botulinum toxin in patients with recurrent acute pancreatitis due to pancreatic sphincter of Oddi dysfunction. Alimentary Pharmacology \&amp; Therapeutics. 2000; 14(11): 1469-77.

[12] Chen C-Y, Liao C-H, Kuo H-C. Therapeutic effects of detrusor botulinum toxin A injection on neurogenic detrusor overactivity in patients with different levels of spinal cord injury and types of detrusor sphincter dyssynergia. Spinal Cord. 2011; 49(5): 659-64.

[13] Orasanu B, Mahajan S. The use of botulinum toxin for the treatment of overactive bladder syndrome. Indian Journal of Urology. 2013; 29(1): 2.

[14] Dressler D, Saberi F, Barbosa E. Botulinum toxin: mechanisms of action. Arquivos de Neuro-Psiquiatria. 2005; 63(1): 180-185.

[15] de Paiva A, Meunier F, Molgo J, Aoki K, Dolly J. Functional repair of motor endplates after botulinum neurotoxin type A poisoning: Biphasic switch of synaptic activity between nerve sprouts and their parent terminals. Proceedings of the National Academy of Sciences. 1999; 96(6): 3200-3205.

[16] Shen J, Ma J, Lee C, Smith B, Smith T, Tan K et al. How muscles recover from paresis and atrophy after intramuscular injection of botulinum toxin A: Study in juvenile rats. Journal of Orthopaedic Research. 2006; 24(5): 1128-1135.

[17] Naumann M, Carruthers A, Carruthers J, Aurora S, Zafonte R, Abu-Shakra S et al. Meta-analysis of neutralizing antibody conversion with onabotulinumtoxinA (BOTOX®) across multiple indications. Movement Disorders. 2010; 25(13): 2211-2218.

[18] Kassir M, Gupta M, Galadari H, Kroumpouzos G, Katsambas A, Lotti T et al. Complications of botulinum toxin and fillers: A narrative review. Journal of Cosmetic Dermatology. 2019; 19(3): 570-573.

[19] Bai L, Peng X, Liu Y, et al. Clinical analysis of 86 botulism cases caused by cosmetic injection of botulinum toxin (BoNT). Medicine (Baltimore). 2018; 97(34): e10659.

[20] Yiannakopoulou E. Serious and Long-Term Adverse Events Associated with the Therapeutic and Cosmetic Use of Botulinum Toxin. Pharmacology. 2015; 95(1-2): 65-69.

[21] Binder W, Brin M, Blitzer A, Pogoda J. Botulinum toxin type A (BOTOX) for treatment of migraine. Disease-aMonth. 2002; 48(5): 323-335.

[22] Dhaked RK, Singh MK, Singh P, Gupta P. Botulinum toxin: bioweapon \& magic drug. Indian J Med Res. 2010; 132(5): 489-503. 